\title{
Magnetized Electron Beam for JLEIC Re-circulator Cooler Ring
}

\author{
M. Poelker ${ }^{1}$, P. Adderley, J. Benesch, B. Bullard, J. Grames, F. Hannon, \\ J. Hansknecht, C. Hernandez-Garcia, R. Kazimi, G. Krafft, M. A. Mamun,
}

R. Suleiman, M. Tiefenback, Y. Wang, S. Wijiethunga, J. Yoskovitz, and S. Zhang

Thomas Jefferson National Accelerator Facility

12000 Jefferson Ave., Newport News, VA 23606 USA

E-mail: poelkerejlab.org

\begin{abstract}
The ion beams of the proposed Jefferson Lab Electron Ion Collider (JLEIC) must be cooled to achieve the required collision luminosity. In general, cooling is accomplished when an electron beam co-propagates with an ion beam moving at the same average velocity, but with different temperature, where the energy of chaotic motion of the ion beam is transferred to the cold electron beam. The cooling rate can be improved by about two orders of magnitude if the process occurs inside a solenoidal magnetic field - so-called magnetized cooling - that forces the electrons to follow small helical trajectories thereby increasing the interaction time with ions and improving the cooling efficiency. However, one of the challenges associated with implementing this cooling technique relates to the fringe field of the cooling solenoid which imparts a large unwanted azimuthal kick onto the electron beam that prevents the electron beam from traveling in the desired tight, well-defined volume within the solenoid. As proposed by Derbenev, the ill-effect of this fringe field can be cancelled if the electron beam is born in a similar field and encountering a fringe field upon exiting the electron gun that produces an azimuthal kick in the opposite direction, such that the two kicks cancel. Besides requiring magnetized beam, the JLEIC re-circulator cooler design requires an electron beam with very high average current and high bunch charge: $140 \mathrm{~mA}$ and with nanoCoulomb bunch charge. This contribution describes the latest milestones of a multiyear program to build a magnetized electron beam source based on a $350 \mathrm{kV}$ DC high voltage photogun with inverted insulator geometry.
\end{abstract}




\section{Introduction}

It is believed that efficient cooling of ion beams at the proposed Electron Ion Collider (EIC) can be achieved using magnetized electron beams with energy of tens of $\mathrm{MeV}$, and at beam currents of a few Amperes. These specifications led to the Jefferson Lab EIC (JLEIC) recirculator cooler design [1] shown in Figure 1, with key features that include long cooling solenoids, energy recovery, beam re-circulation and fast kickers to refresh spent electron bunches. The multi-pass re-circulator ring serves to reduce the burden on the electron source by a factor equal to the number of re-circulation passes, presently assumed to be of the order ten. However, even with this design feature, the electron gun must still provide $140 \mathrm{~mA}$ average current and with $3.2 \mathrm{nC}$ bunch charge. These specifications are well beyond today's state of the art (Table 1) [2]. This submission describes a multi-year effort to construct a $350 \mathrm{kV}$ DC high voltage photogun that relies on robust alkali-antimonide photocathodes, and to demonstrate the production of magnetized electron beams at very high average current and bunch charge.

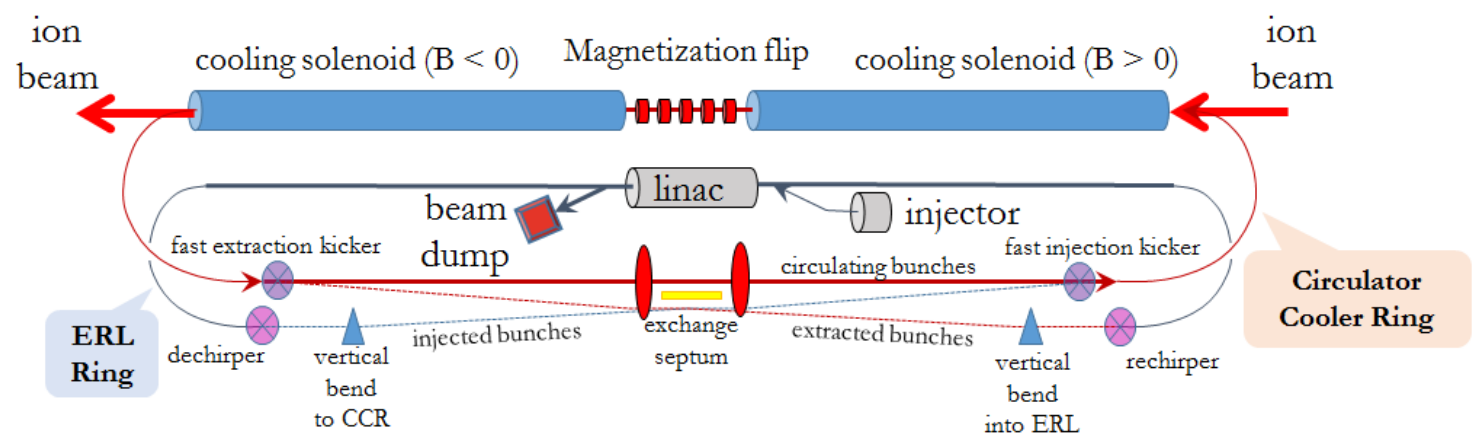

Figure 1: A schematic of the JLEIC re-circulator cooler that employs long cooling solenoids, a re-circulator ring to reduce the current requirement on the photogun, energy recovery and fast kickers to replace spent electron bunches.

Table 1. The electron beam requirements of JLEIC re-circulator cooler design compared to relevant state-of-the-art electron beam demonstrations

\begin{tabular}{|l|c|c|c|}
\hline & $\begin{array}{c}\text { JLEIC magnetized } \\
\text { beam requirements }\end{array}$ & $\begin{array}{c}\text { Cornell } \\
\text { University }\end{array}$ & $\begin{array}{c}\text { Fermi Lab } \\
\text { ASTA Facility }\end{array}$ \\
\hline Bunch charge $[\mathrm{nC}]$ & 3.2 & 0.05 & 0.5 \\
\hline Average current $[\mathrm{mA}]$ & 140 & 65 & 0.0075 \\
\hline Repetition rate $[\mathrm{MHz}]$ & 43.3 & 1300 & $\begin{array}{c}3 \\
(0.5 \% \text { duty factor })\end{array}$ \\
\hline Bunch length $[\mathrm{ps}]$ & 60 & 3 & 3 \\
\hline $\begin{array}{l}\text { Transverse normalized emittance } \\
\text { [mm-mrad] }\end{array}$ & $<19$ & 0.4 & 4 \\
\hline Beam Magnetization? & Yes & No & Yes \\
\hline
\end{tabular}

As described in the abstract, one of the challenges to implementing magnetized electron beam cooling is delivering the electron beam into the cooling solenoids while maintaining a well-confined beam envelope that overlaps the ion beam, with electrons traveling in tight helical 
trajectories. The challenge associated with injecting the electron beam into the cooling solenoids stems from the Lorentz force associated with the transverse component of the solenoid magnet fringe field which produces a large azimuthal deflection on the electron beam, causing large electron transverse motion and preventing the overlap of the electron and ion beams. To address this issue, Derbenev [3] proposed a solution: have the electron beam originate within a solenoidal magnetic field and upon exiting the electron gun, the beam will experience a fringe field that produces an azimuthal kick in the opposite direction, such that the two azimuthal deflections cancel. There have been successful demonstrations of magnetized beam production, but only with a pulsed-RF photogun at low average current [4].

A number of dc high voltage photoguns rely on large cylindrical ceramic insulators to electrically isolate the cathode electrode [5-7] which must be supported on a long coaxial metal support structure. In contrast to these designs, an inverted-insulator geometry design was chosen at Jefferson Lab for two reasons: first to provide a smaller volume which could result in better achievable vacuum because there is less surface area to contribute a gas load; and second, the insulator serves as the electrode support structure which means there is less metal biased at high voltage, and less metal to contribute to field emission. The photogun described in this work is a larger version of the $130 \mathrm{kV}$ dc high voltage photogun used at the Continuous Electron Beam Accelerator Facility (CEBAF) [8]. The cylindrical vacuum chamber design shown in Figure 2 is relatively compact, $45 \mathrm{~cm}$ diameter, and with volume and surface area approximately one third of photogun designs with large cylindrical insulators that operate at comparable voltage. The cathode electrode includes a screening electrode (the doughnut-shaped ring above the spherical electrode, Figure 2 right) that serves to significantly reduce the field strength at the delicate triple point, where insulator, metal and vacuum meet [9]. The photogun was kryptongas conditioned to $360 \mathrm{kV}$ and biased at $300 \mathrm{kV}$ for the beam studies described here. Operation at the intended $350 \mathrm{kV}$ will occur following the completion of commissioning studies.

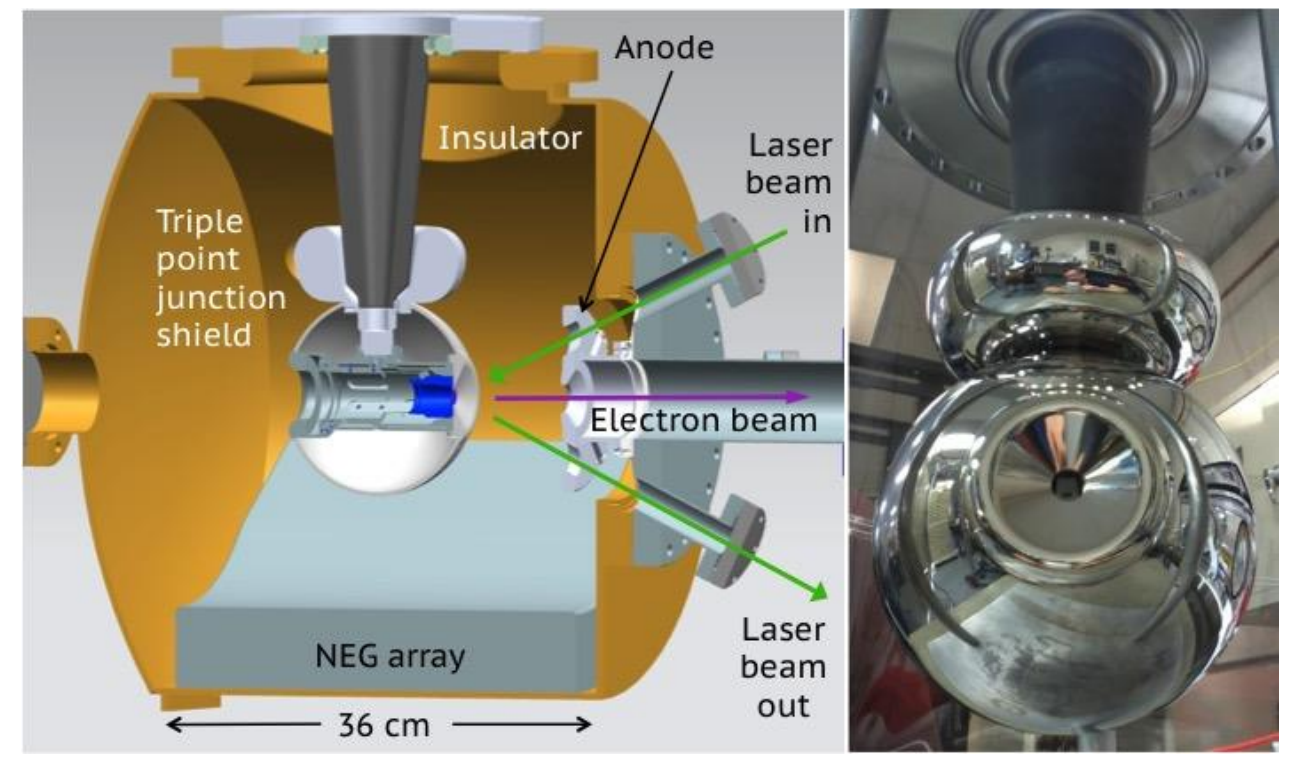

Figure 2: a) Cross-section view of the $350 \mathrm{kV}$ dc high voltage photogun with inverted insulator geometry. The photocathode (purple) and puck (blue) rest inside the $15.25 \mathrm{~cm}$ diameter spherical cathode electrode with $25^{\circ}$ Pierce focusing angle. b) Photograph of the stainless steel spherical electrode attached to the doped-alumina ceramic insulator welded to a $25 \mathrm{~cm}$ Conflat flange. 
Alkali-antimonide photocathodes $\left(\mathrm{Cs}_{\mathrm{x}} \mathrm{K}_{\mathrm{y}} \mathrm{Sb}\right)$ are grown on $\mathrm{GaAs}$ substrates attached to molybdenum "pucks" inside the deposition chamber located behind the photogun (Figure 3). Once fabricated, the photocathode can be inserted into the photogun cathode electrode using a magnetic sample manipulator. A solenoid magnet located immediately downstream of the anode of the photogun can provide magnetic fields at the photocathode up to $1.5 \mathrm{kG}$, and with a bore sufficiently large to enable illumination of the photocathode with laser light incident at a relatively small angle without requiring turning mirrors placed inside the beamline vacuum chamber. The electron beam is delivered to a $2 \mathrm{~kW}$ beam dump approximately $4 \mathrm{~m}$ away, using solenoid magnets to focus the beam, and steering magnets to center the beam within the vacuum chamber and on beamline viewers. One viewer cross includes a narrow tungsten slit designed to pass a narrow beamlette used to evaluate beam magnetization that manifests itself as a rotation of the narrow beam slice on downstream viewers.

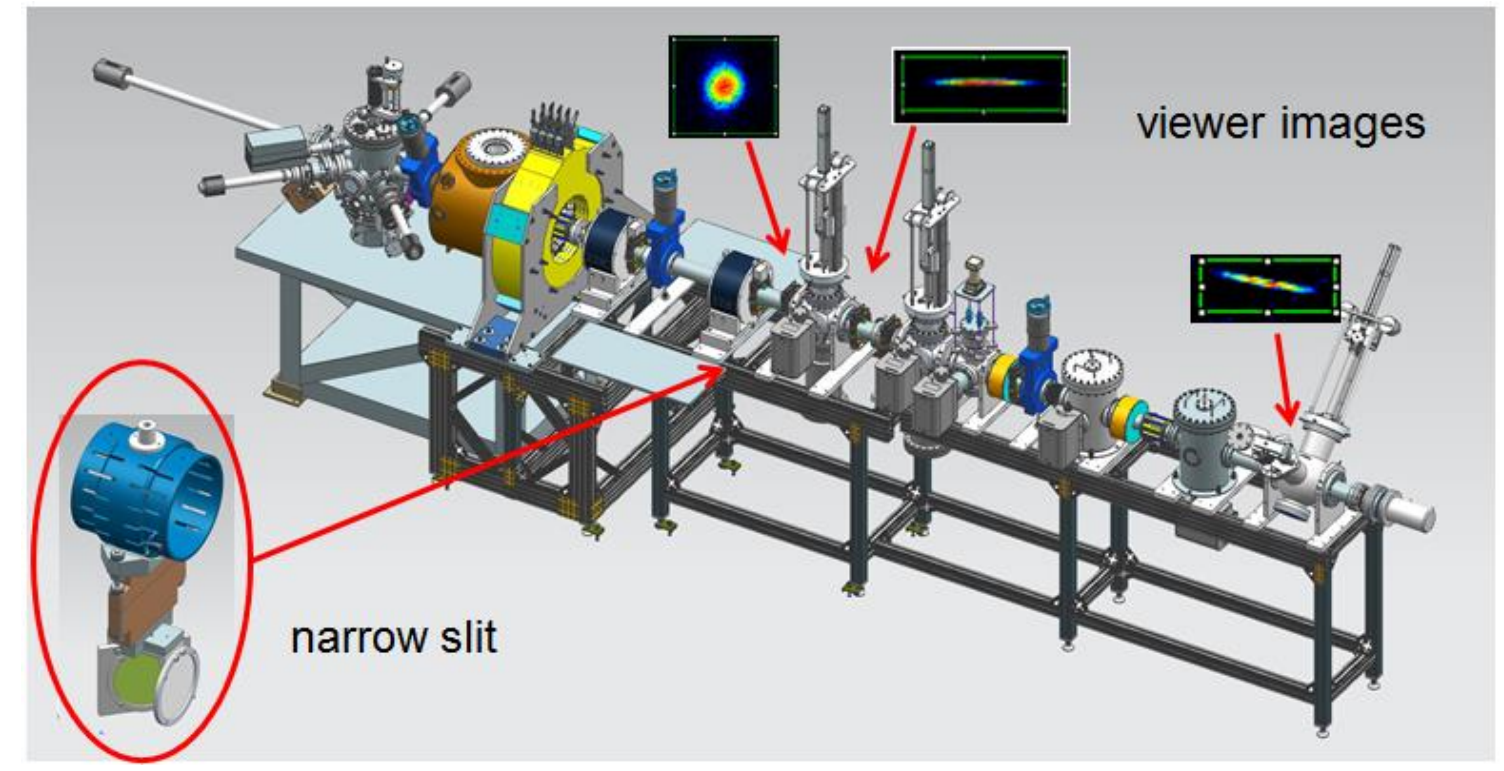

Figure 3: The magnetized-beam test apparatus includes (from left to right) the alkali-antimonide photocathode deposition chamber, the dc high voltage photogun, the magnetization solenoid, and diagnostic beamline with a slit that passes a narrow beam slice that can be viewed on downstream viewers to evaluate beam rotation and magnetization, as shown in the insets.

The beam's magnetization, or mechanical angular momentum $\langle L\rangle$, can be set to a specific value by adjusting the magnetic field, $B_{z}$, and laser beam diameter, $a_{0}$, at the photocathode as shown in the equation below [4]:

$$
\langle L\rangle=2 p_{z} \frac{\sigma_{1} \sigma_{2} \sin \varphi}{D}=e B_{z} a_{0}^{2}
$$

Equation 1 also describes how the beam magnetization can be verified experimentally, by creating a narrow beam slice using the slit and delivering this beam to a downstream viewer, where the rotation angle $\varphi$ can be measured. Quantities $p_{z}, \sigma_{1}, \sigma_{2}$ and $D$ represent the beam momentum, the size of the beam at the slit and at a downstream viewer, and the drift distance between the slit and viewer, respectively. 


\subsection{Results}

Magnetized beam was passed through the narrow slit and delivered to a downstream viewer at a drift distance of $2.3 \mathrm{~m}$. The rotation of the beam slice was measured as a function of gun solenoid field strength, with results shown in Figure 4. Rotation oscillations were observed as a result of recurring beam focusing caused by the relatively long gun solenoid, where peak rotation values correspond to tight beam waists produced at the location of the viewer.

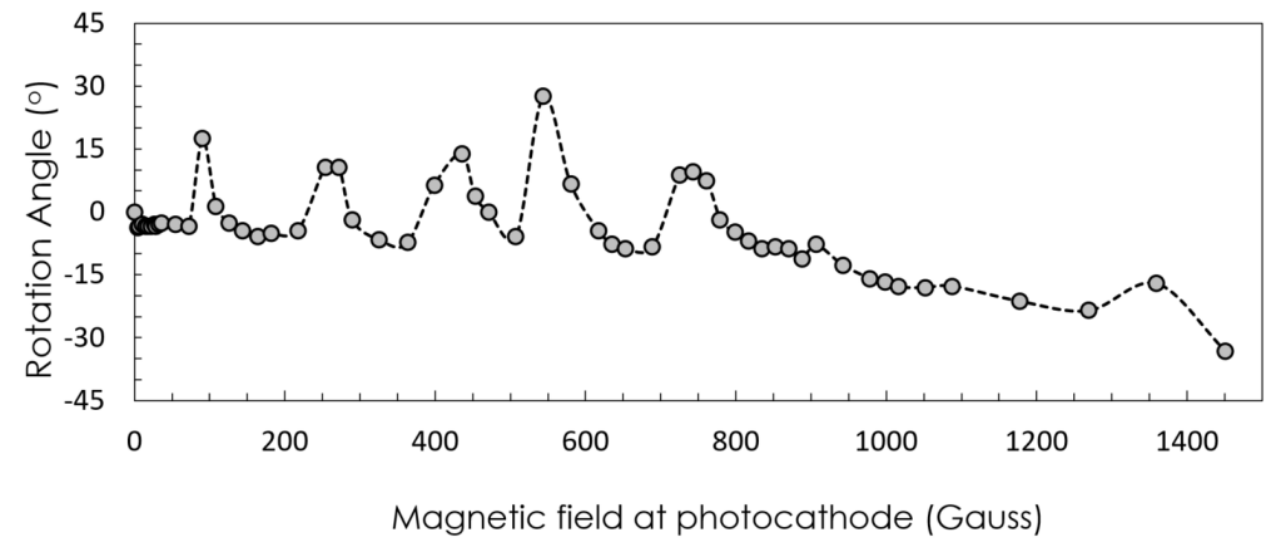

Figure 4: The measured rotation of a magnetized beam slice delivered to a downstream viewer at $2.3 \mathrm{~m}$ drift distance on the diagnostic beam line, as a function of gun solenoid magnetic field.

Finally, with the slit and viewers removed from the beamline, high average current magnetized beam at $4.5 \mathrm{~mA}$ was delivered to the dump. Initial measurements indicate a photocathode lifetime of approximately 160 Coulombs, where "lifetime" describes the amount of charge that could be extracted before photocathode quantum efficiency fell to $1 / \mathrm{e}$ of the initial value.

\subsubsection{Conclusions}

These measurements represent the first demonstrations of magnetized beam produced using a dc high voltage photogun at $300 \mathrm{kV}$ bias voltage, and at milliAmpere average current. Admittedly, the photocathode lifetimes measured at Jefferson Lab were not as good as reported elsewhere, using similar photocathode material, and this will be the focus of study in the near future. Implementing a biased anode, beamline ion precipitators, masked photocathode active area that is offset from the electrostatic center, metallic substrates and alternative photocathode recipes may be required to improve photocathode lifetime.

Particle tracking code simulations are being developed to predict the beam envelope along the beamline, beam magnetization and the observed rotation angles as a function of gun solenoid field strength. An accurate model of the photogun and beamline will assist the production of magnetized beam at high bunch charge. More detailed results will be presented in a future publication. 


\section{References}

[1] S. Benson, Ya. Derbenev, D. Douglas, F. Hannon, A. Hutton, R. Li, R. Rimmer, Y. Roblin, C. Tennant, H. Wang, H. Zhang, Y. Zhang, "Development of a bunched-beam electron cooler based on ERL and Circulator Ring technology for the Jefferson Lab Electron-Ion Collider", 11th Workshop on Beam Cooling and Related Topics, COOL2017, Bonn, Germany, JACoW Publishing ISBN: 978-3-95450-198-4, doi: 10.18429/JACoWCOOL2017-WEM12

[2] B. Dunham, A. Bartnik, I. Bazarov, L. Cultrera, J. Dobbins, G. Hoffstaetter, B. Johnson, R. Kaplan, V. Kostroun, S. Karkare, Y. Li, M. Liepe, X. Liu, F. Loehl, J. Maxson, P. Quigley, J. Reilly, D. Rice, D. Sobol, E. Smith, K. Smolenski, M. Tigner, V. Veshcherevich, Z. Zhao, "Record high-average current from a high-brightness photoinjector", Appl. Phys. Lett. 102, 034105 (2013)

[3] Ya. Derbenev, “Advanced optical concepts for electron cooling”, Nucl. Inst. Meth. A 441 223 (2000); Ya. Derbenev, “Adapting optics for high energy electron cooling”, University of Michigan Report No. UM-HE-98-04, (1998)

[4] Y.-E Sun, P. Piot, K.-J. Kim, N. Barov, S. Lidia, J. Santucci, R. Tikhoplav, and J. Wennerberg, "Generation of angular-momentum dominated electron beams from a photoinjector", Phys. Rev. ST Accel. Beams, Vol. 7, 123501 (2004)

[5] J. Maxon, I. Bazarov, B. Dunham, J. Dobbins, X.Liu, and K. Smolenski, "Design, conditioning, and performance of a high voltage, high brightness dc photoelectron gun with variable gap", Rev. Sci, Instrum. Vol. 85, 093306, 2014

[6] C. Hernandez-Garcia, T. siggins, S. Benson, D. Bullard, H. F. Dylla, K. Jordan, C. Murray, G. R. Neil, M. Shinn, and R. Walker, "A high average current dc GaAs photocathde gun for ERLs and FELs", Proceedings of the 2005 Particle Accelerator Conference, Knowxville, TN, USA, pp. 3117-3119 (2005)

[7] N. Nishimori, R. Nagai, S. Matsuba, R. Hajima, M. Yamamoto, Y.Honda, and T. Miyajima, "Experimental investigation of an optimum configuration for a high-voltage photoemission gun for operation at > $500 \mathrm{kV}$ ", Phys. Rev. ST Accel. Beams, Vol. 17, 053401 (2014)

[8] P. A. Adderley, J. Clark, J. Grames, J. Hansknecht, K. Surles-Law, D. Machie, M. Poelker, M. L. Stutzman, and R. Suleiman, "Load-locked dc high voltage GaAs photogun with an inverted-geoemtry ceramic insulator", Phys. Rev. ST Accel. Beams 13, 010101 (2010)

[9] C. Hernandez-Garcia, D. Bullard, F. Hannon, Y. Wang and M. Poelker, "High voltage performance of a dc photoemission electron gun with centrifugal barrel-polished electrodes", Rev. Sci. Instrum. 88, 093303 (2017) 